\title{
The stability of vacuum phototriodes to varying light pulse loads and long term changes in response
}

\author{
Peter R Hobson ${ }^{1^{*}}$ \\ School of Engineering \& Design, Brunel University, Uxbridge, UB8 3PH, UK \\ E-mail: Peter.Hobson@brunel.ac.uk
}

\begin{abstract}
Mesh anode Vacuum Phototriodes (VPTs) are radiation resistant, single gain-stage photomultipliers which are designed to operate in a strong quasi-axial magnetic field. These VPTs are used in the endcap electromagnetic calorimeter of the CMS experiment at the CERN LHC to detect scintillation light from lead tungstate crystals. Short term dynamic response changes occur because of pulse rate variations during normal LHC operation cycles. Over the longer term the effect of increasing integrated charge taken from the photocathode causes an overall degradation of response. We have investigated these effects over time periods exceeding two years of simulated operation and discuss the implications for the long term performance of the VPTs in CMS.
\end{abstract}

International Workshop on New Photon-detectors,

LAL Orsay, France

June 13-15, 2012

Speaker

For the CMS Collaboration 


\section{Introduction}

The CMS experiment [1] at the Large Hadron Collider (LHC), CERN must operate for many years in an environment in which the detector subsystems will degrade due to a combination of operational life and radiation damage. The two electromagnetic calorimeter endcaps (EE) are constructed from four half-disk 'dees', each 'dee' consisting of 3662 tapered lead tungstate scintillation crystals, arranged in a quasi-projective geometry. The scintillation light is detected by mesh anode vacuum phototriodes (VPTs) which operate at gains of approximately 10 in a $3.8 \mathrm{~T}$ solenoidal magnetic field. The design and construction of the EE, the readout electronics and the performance of individual system components is described elsewhere [2].

The VPT type PMT188 is a single gain-stage photomultiplier which can operate in high quasi-axial magnetic fields and is produced by National Research Institute Electron [3]. It has an outer diameter of $26 \mathrm{~mm}$ and active area of $280 \mathrm{~mm}^{2}$. The development and evaluation of prototype VPTs is described elsewhere [4,5].

The VPT response slowly degrades with time due to ionizing radiation damage to the faceplate on which the photocathode is deposited [6] and to the well known loss of response in vacuum photodetectors, assumed to be due to damage from ion feedback, as charge is taken from the device [7]. In addition there are dynamic rate-dependent effects where short term changes in response are induced by significant changes in the rate of light pulses incident upon the device. Previous work [2] has already revealed a small percentage change in VPT response when the rate changes significantly. This needs to be understood and, if possible, controlled so that these short-term variations do not contribute significantly to the systematic error of the energies measured in the EE.

\section{Pulse rate and long term charge related effects on VPT response}

VPTs have been seen to exhibit short-term changes in response which are related to the average current being drawn. To minimise these effects during LHC beam-on and beam-off cycles an LED pulser system in the EE, comprising blue $(440 \mathrm{~nm})$ and orange $(617 \mathrm{~nm})$ LEDs is provided. This two-colour LED system also allows us to monitor separately radiation damage to lead tungstate transparency and VPT photocathode degradation effects. Similarly a gradual overall decrease in response, related to the total charge extracted from the VPT, is expected to be seen as the LHC integrated luminosity increases with time.

\subsection{Rate dependent effects}

Measurements made at a CERN test beam (figure 1) alerted us to a rate effect at 0T which gave transient gain changes of $\sim 5 \%$. This rate-dependent effect is important at the start of an LHC fill. Operating the VPT at the nominal CMS field of 3.8T resulted in the amplitude of these effects being reduced below $0.5 \%$. 


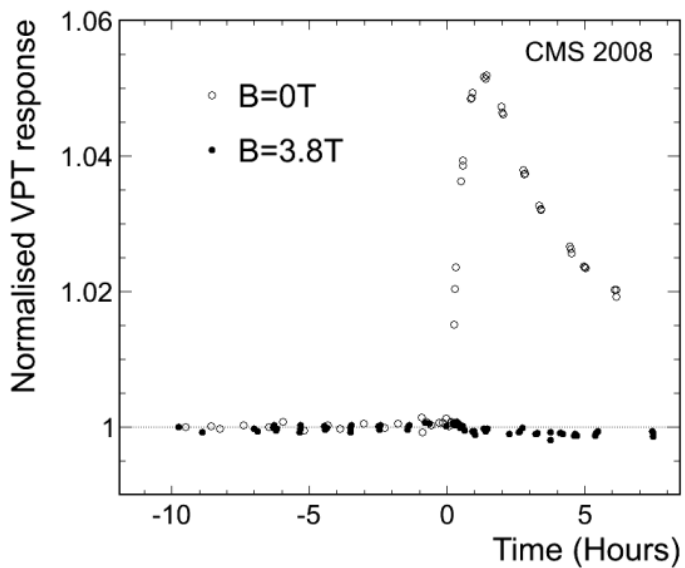

Figure 1 Average normalised response of 200 VPT operating in a field of 0 T (open circles) and $3.8 T$ (filled circles). In both tests LED pulsing with a rate of $10 \mathrm{kHz}$ was performed for a period of 17 hours and turned off at the point $T=0$ hours.

The normalised response of a VPT operating at an angle of $15^{\circ}$ to a $4 \mathrm{~T}$ field is shown in figure 2. An LED pulsing with a rate of $20 \mathrm{kHz}$ was on for 20 hours then off for 4 hours daily. The 1nA average photocathode current, when the LED is on, is appropriate for a VPT located at $\eta=2.1$ in the EE and an LHC peak luminosity of $10^{34} \mathrm{~cm}^{-2} \mathrm{~s}^{-1}$.

The VPT response was measured with a separate stable LED operating at $10 \mathrm{~Hz}$. The change in response with rate can be clearly seen, but its peak magnitude is below $\pm 0.5 \%$

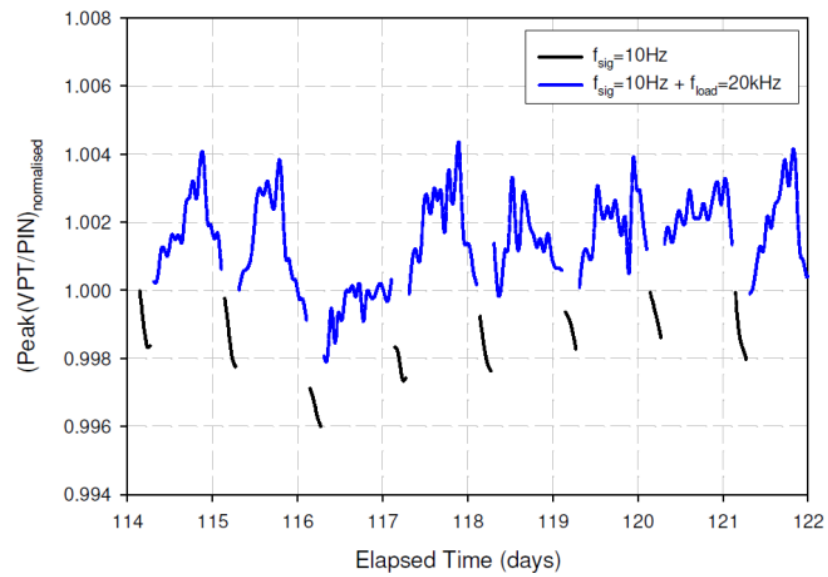

Figure 2 Response of a VPT in a 4 T field. A load LED pulsing with a rate of $20 \mathrm{kHz}$ was on for 20 hours then off for 4 hours daily. The response of the VPT is normalised to that of a reference pin photodiode.

The improvement in VPT stability is ascribed to the minimisation, at 4T, of the influence on electron trajectories of mobile charges on the insulating surfaces within the VPT envelope.

\subsection{Total charge extracted effect on response}

The long term stability of the VPTs was studied by exposing the photocathode to blue LED light. Figure 3 shows the relative VPT response versus integrated charge drawn from the photocathode; an extracted photocathode charge of $10 \mathrm{mC}$ corresponds to an integrated LHC luminosity of $40 \mathrm{fb}^{-1}$ at $\eta=2.5$ in the EE. This VPT had already provided an initial integrated charge of appoximately $0.6 \mathrm{mC}$ before the measurements reported here. 


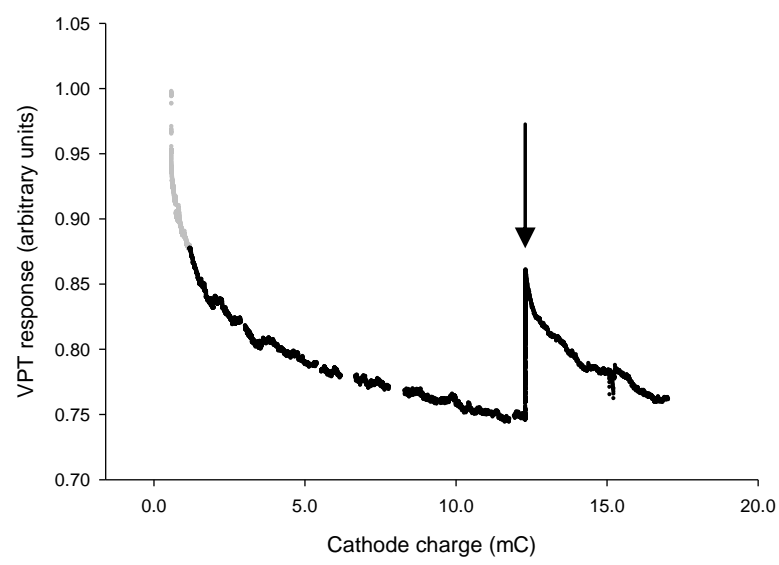

Figure 3 Change in response versus extracted cathode charge for a VPT operating in a 4T field. The grey data points correspond to an average cathode current of $0.25 n A$, black data points to 1 nA. At the point indicated by the arrow the average current was reduced to less than 1 PA for a period of twelve months before being increased again to $1 \mathrm{nA}$.

\section{Summary}

VPTs show both short term rate-dependent effects and a long term reduction in response which is a function of photocathode charge delivered. The short term variations are reduced to acceptable levels $(<0.5 \%)$ when the VPTs operate at the CMS field of $3.8 \mathrm{~T}$.

Long term high current operation causes losses which appear to reach a plateau. Measurements indicate that these are primarily associated with a reduction in photocathode quantum efficiency rather than any change in dynode gain. Plateau values vary with VPT between $\sim 2 \%$ decrease in original response and $\sim 35 \%$ (sample size $=14$ ). These losses partially recover with time at very low cathode currents. No increase in the noise of a VPT operating continuously at $4 \mathrm{~T}$ for over 2 years was observed.

\section{References}

[1] CMS Collaboration, The CMS Experiment at the CERN LHC, JINST 3 (2008) S08004

[2] CMS Collaboration, Performance and operation of the CMS electromagnetic calorimeter, JINST 5 (2010) T03010

[3] Toreza ave. 68, 194223, St. Petersburg, Russia

[4] K.W. Bell et al, The development of vacuum phototriodes for the CMS electromagnetic calorimeter, Nuclear Instruments and Methods in Physics Research A 469 (2001) 29-46

[5] M. Apollonio et al, The performance of prototype vacuum phototriodes in the first full sized supercrystal array for the CMS ECAL endcaps, Nuclear Instruments and Methods in Physics Research A 532 (2004) 566-574

[6] K.W. Bell et al, Vacuum phototriodes for the CMS electromagnetic calorimeter endcap, IEEE Transactions on Nuclear Science 51 (2004) 2284-2287

[7] J.P. Boutot, J. Nussli, D. Vallat, Recent Trends in Photomultipliers for Nuclear Physics, Advances in Electronics and Electron Physics 60 (1983) 223-305 